\title{
THE HIGH FLUX BEAM REACTOR AT BROOKHAVEN NATIONAL LABORATORY
}

\author{
S.M. SHAPIRO \\ Dept. of Physics, Brookhaven National Laboratory, Upton, NY 11973, USA
}

\section{HIGH FLUX BEAM REACTOR}

\begin{abstract}
Brookhaven National Laboratory's High Flux Beam Reactor (HFBR) was built because of the need of the scientist to always want 'more'. In the mid-50's the Brookhaven Graphite reactor was churning away producing a number of new results when the current generation of scientists, led by Donald Hughes, realized the need for a high flux reactor and started down the political, scientific and engineering path that led to the HFBR. The effort was joined by a number of engineers and scientists among them, Chernick, Hastings, Kouts, and Hendrie, who came up with the novel design of the HFBR. The two innovative features that have been incorporated in nearly all other research reactors built since are: (i) an under moderated core arrangement which enables the thermal flux to peak outside the core region where beam tubes can be placed, and (ii) beam tubes that are tangential to the core which decrease the fast neutron background without affecting the thermal beam intensity. Construction began in the fall of 1961 and four years later, at a cost of $\$ 12$ Million, criticality was achieved on Halloween Night, 1965. Thus began 30 years of scientific accomplishments.
\end{abstract}

\section{Reactor}

The HFBR uses highly enriched U-235 fuel and heavy water to moderate and cool the reactor. The core consists of 28 fuel elements, each containing 18 curved fuel plates. In each plate, $\mathrm{U}_{3} \mathrm{O}_{8}$ powder is mixed with aluminum powder to form a cermet core which is encased in aluminum cladding. The fuel elements are placed in a roughly cylindrical arrangement inside a spherical-shaped reactor vessel. The core is approximately $53 \mathrm{~cm}$ high and $48 \mathrm{~cm}$ in diameter and contains a maximum of $9.8 \mathrm{~kg}$ of $\mathrm{U}-235$. The reactor and its equipment, plus the experimental facilities are contained in a welded steel hemisphere $53.6 \mathrm{~m}$ in diameter. The experimental level containing all the instrumentation is separated from the operations and equipment level as shown in Fig. 1. The reactor operated at $40 \mathrm{MW}$ for 17 years and in 1982, the heat exchangers were replaced and the power increased to $60 \mathrm{MW}$, producing a peak thermal flux of $1 \times 10^{15} \mathrm{n} / \mathrm{cm}^{2}-\mathrm{sec}$ at about $30 \mathrm{~cm}$. from the core center, where the beam tubes are placed (Fig. 2).

The HFBR has had a reliable history and has operated over 250 cycles of 3-4 weeks duration since it first went critical. Unfortunately in April, 1989 it was forced into a prolonged shut down for a safety review. After extensive studies, the reactor was restarted in May, 1991. The permitted power is only $30 \mathrm{MW}$, one-half of normal, until further thermal hydraulic testing. The calculations and tests have been completed and analyzed and will be used as a basis for a request to resume higher power operation.

\section{DISCLAIMER}

This report was prepared as an account of work sponsored by an agency of the United States Government. Neither the United States Government nor any agency thereof, nor any of their employees, makes any warranty, express or implied, or assumes any legal liability or responsibility for the accuracy, completeness, or usefulness of any information, apparatus, product, or process disclosed, or represents that its use would not infringe privately owned rights. Reference herein to any specific commercial product, process, or service by trade name, trademark, manufacturer, or otherwise does not necessarily constitute or imply its endorsement, recommendation, or favoring by the United States Government or any agency thereof. The views and opinions of authors expressed herein do not necessarily state or reflect those of the United States Government or any agency thereof. 


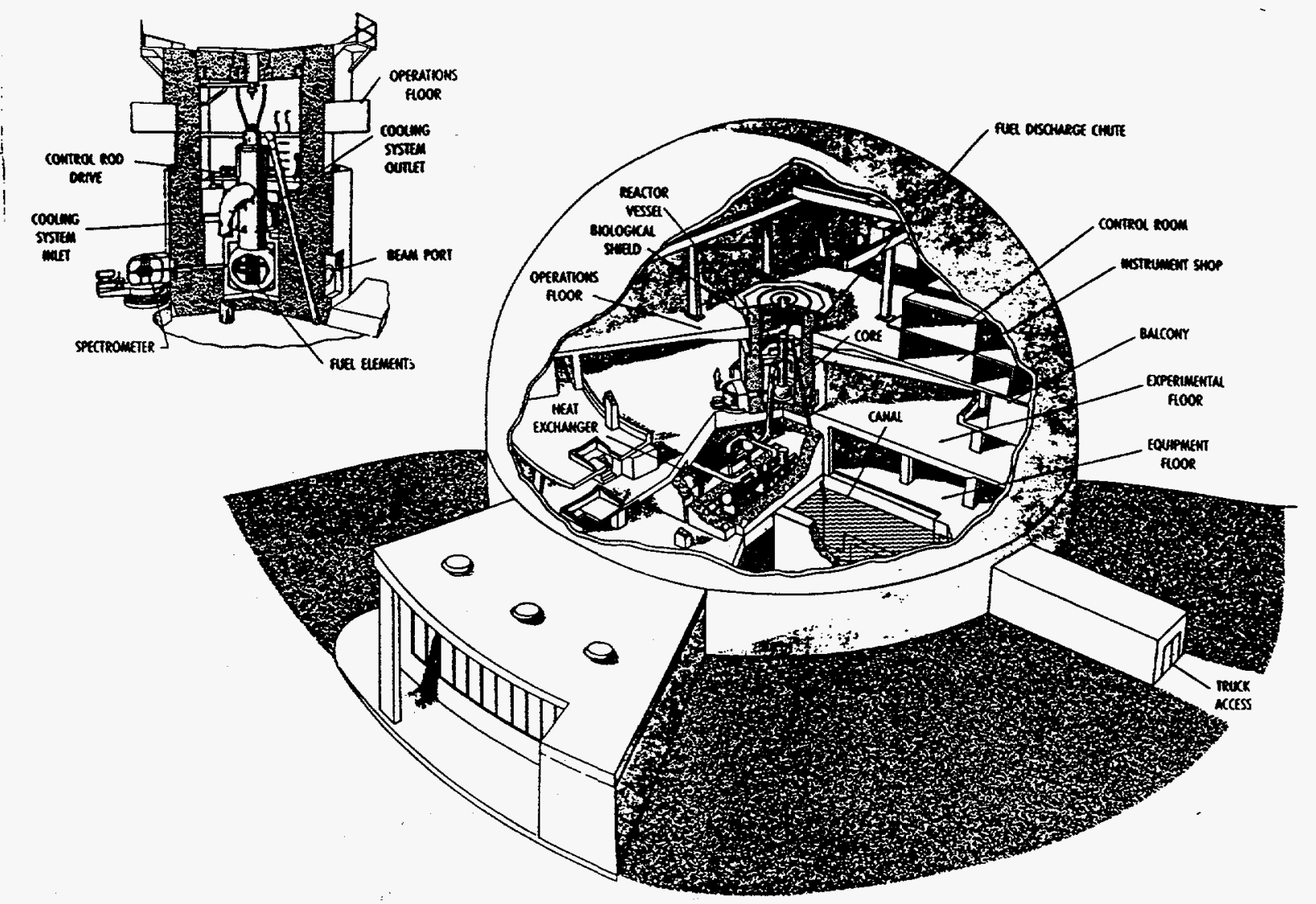

Fig. 1 Cut-away view of the HFBR building showing the arrangement of the building and a close up of the reactor (upper left).

$\underline{\text { Beams }}$

There are eight tangential and one radial beam tube where neutrons emerge from the reactor and are available for experimenters, as shown in the floor plan in Fig. 2. One of the beam tubes (H9) has a larger diameter than the others. A cold neutron moderator, consisting of 1.4 liters of liquid hydrogen was placed in this beam plug and became operational in 1980 . Many of the beam tubes can accommodate more than one experimental facility and there are a total of 15 neutron instruments situated at the HFBR. In addition to the horizontal beam tubes, there are 7 vertical thimbles used for sample irradiation. Three are located in the reflector portion of the reactor, two are at the edge of the core, and two are at the center of the core. 


\section{DISCLAIMER}

Portions of this document may be illegible in electronic image products. Images are produced from the best available original document. 


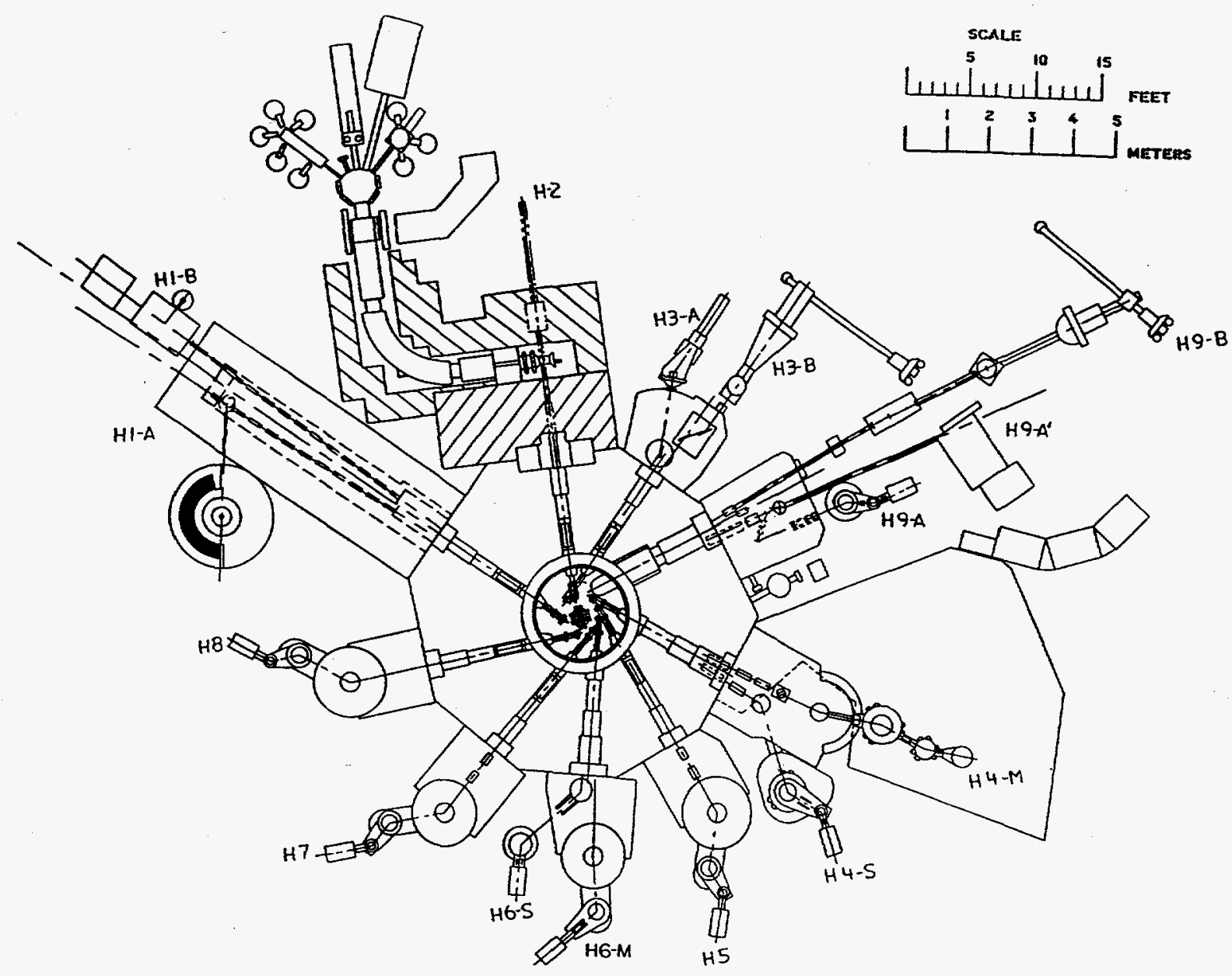

Fig. 2 Layout of instruments on the experimental floor of the HFBR.

\section{Existing Instruments}

The instruments available at the HFBR have been developed to cover a wide range of scientific studies which include structural biology, nuclear physics, structural chemistry, condensed matter physics, and materials science. The layout of the instruments on the reactor floor is shown in Fig. 2. I shall discuss these instruments and give examples of some of the science being done at the HFBR.

\section{Condensed Matter Physics and Materials Science:}

(H4M, H7, H8, H9-A (cold source)): Condensed matter studies use mainly 3-axis instruments which are operated in the elastic or inelastic mode. One 3-axis instrument is situated on the cold neutron moderator which allows for higher energy and momentum resolution. The thermal instruments have a flux at the monochromators position (with $20 \mathrm{~min}$. collimation) of $4 \times 10^{9}$ $\mathrm{n} / \mathrm{cm}^{2} / \mathrm{sec}$. under normal $60 \mathrm{MW}$ of power. (Hereafter, all flux levels will be quoted for $60 \mathrm{MW}$ ). All instruments are equipped with focusing pyrolytic graphite (PG) monochromator and have pneumatically controlled lifters inside the monochromators shielding to move a PG or Be filter into and out of the beam. Some Be monochromators are also available and can be interchanged for the PG monochromator. Collimators are easily interchangeable throughout the instrument to allow the experimenter to easily vary the instrumental resolution. The beam size 
is typically $5 \times 3 \mathrm{~cm}^{2}$. Most instruments have cantilevered analyzer and detector arms, but the newest three axis machine, H4M, moves on air pads floating on a "tanzboden." Polarizing Heusler crystals are available for monochromator and analyzer, as well as flippers and guide fields, so H4M can be operated as a fully polarized beam instrument. This machine was constructed under the international collaboration between the U. S. and Japan on neutron scattering. The three axis instrument on the cold source (H9-A) uses a double monochromator and provides access to the subthermal energy regime.

A major scientific effort of the condensed matter group has been the study of structural and magnetic phase transitions in solids. More recent studies have focused on precursor effects in martensitic transformations and the magnetic and structural properties of high temperature superconductors. Figure 3 shows the results of the low energy portion of the dispersion curve measured for $\mathrm{LaxSr}_{1-\mathrm{x}} \mathrm{CuO}_{4}$. Most of the phonon branches are temperature independent

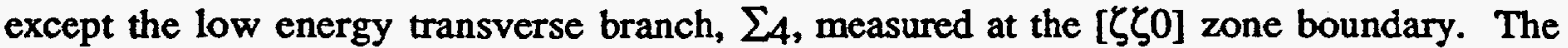
frequency of this mode decreases dramatically and appears to go to zero at the structural transition temperature where the symmetry changes from tetragonal to orthorhombic. The eigenvectors of this mode correspond to a tilting of $\mathrm{CuO}_{6}$ octahedra and are just those required to transform the system from the tetragonal to the orthorhombic phase. Both the superconducting and non-superconducting samples exhibit a similar structural transformation and the role this lattice instability plays in the appearance of superconductivity is still being explored.

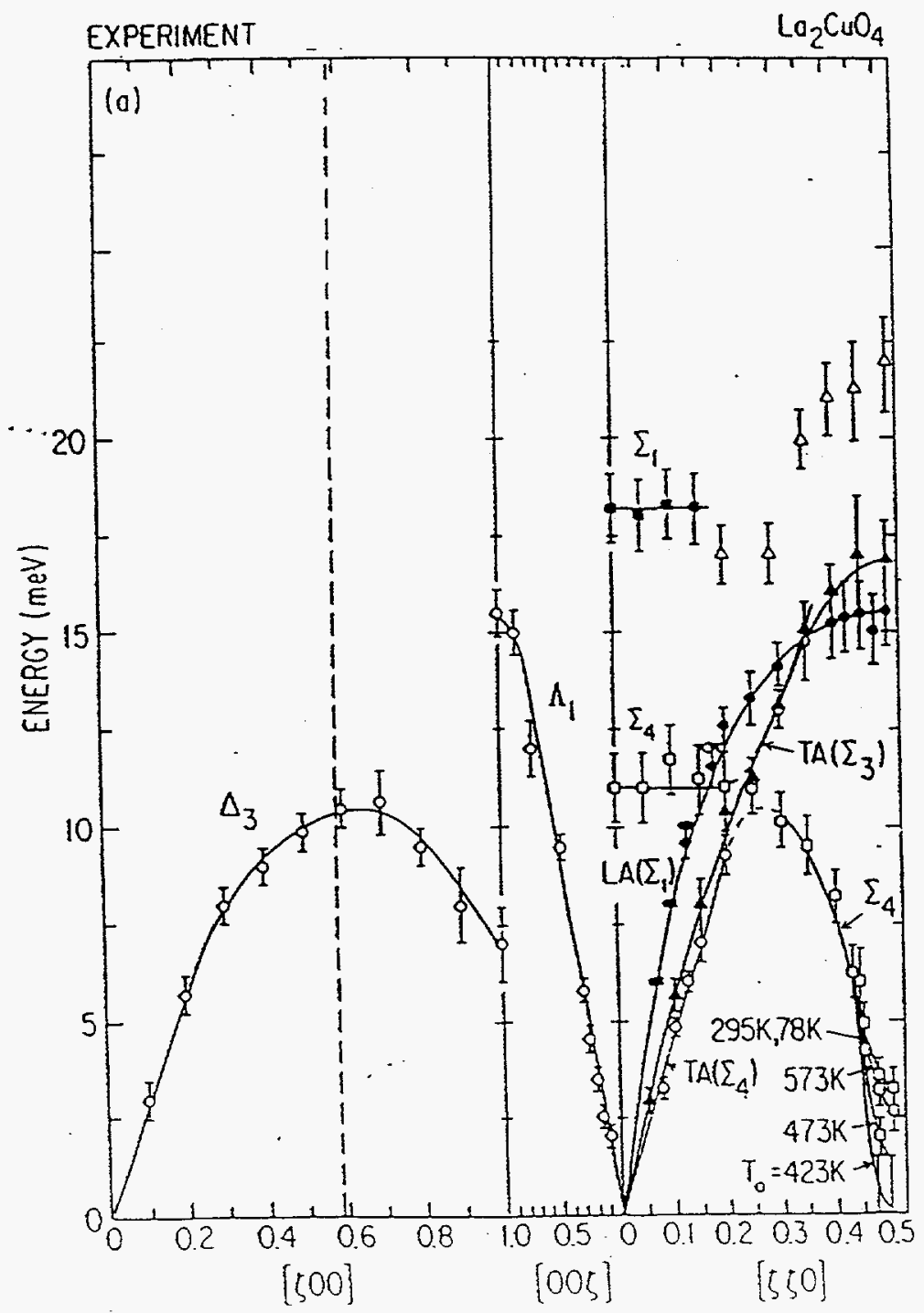

Fig. 3 Low energy (0-25meV) phonon measured in La2$\mathrm{SSr}_{\mathrm{x}} \mathrm{Cu}$ 04. The dispersion curves are only weakly temperature dependent with the exception of the $\Sigma 4$ branch near the $(1 / 2,1 / 2,0)$ zone boundary. (from P. Boni et al., Phys Rev. $\mathrm{B}, 38,185(1988))$. 
Material Science (H4-S, H5): Both H4-S and H-S are triple axis instruments which can operate in the inelastic or elastic modes. H4-S has a fixed scattering angle of the monochromater of $2 \theta \mathrm{M}=41.0^{\circ}$ and by use of $\mathrm{PG}(002)$ or $\mathrm{Si}(311)$ neutrons of wavelengths 2.35 $\AA$ or $1.36 \AA$ can be selected. The two monochromators are situated in a cavity and can be interchanged with each other by a push of a button. H5 is fully automated for variable incident neutron energies and is normally equipped with a focussing PG(002) monochromater, but $\mathrm{Be}$ and $\mathrm{Ge}$ monochromators are available. Each of these instruments can be converted to a high intensity, moderate resolution powder diffractometer, by removing the analyzer and detector arm and replacing it with a housing containing several individual $\mathrm{He}^{3}$ counters. On $\mathrm{H} 5$ there are 15 detectors with $20 \mathrm{~min}$. collimation before each counter. The blades of the collimators are made of stretched mylar films coated with neutron absorbing Gd. The entire detector bank covers an angle of $90^{\circ}$ and each counter is separated by $6^{\circ}$. The maximum two-theta is $135^{\circ}$. $\mathrm{H} 4-\mathrm{S}$ is a smaller version and the detector bank contains 10 counters, each with a 20 min collimation and covers a range of $20^{\circ}$.

Figure 4 is an example of recent measurements on $\mathrm{H}-5$. It is a difference spectrum from a 0.7 monolayer of acetylene $\left(C_{2} D_{2}\right)$ adsorbed on the basal plane of a graphite substrate measured at $\mathrm{T}=10.0 \mathrm{~K}$. Only 15 hours of counting time were required to collect this data. The three diffraction peaks observed are well fit to a square solid structure in which the $\mathrm{C}_{2} \mathrm{D}_{2}$ molecules are oriented at $90 \pm 3^{\circ}$ with respect to one another. The near neighbor distance is $4.325 \AA$. The solid film that forms is incommensurate with the underlying graphite substrate. This lattice structure is consistent with the large quadrupole moment associated with the $\mathrm{C}_{2} \mathrm{D}_{2}$ molecule and agrees well with the results of computer simulation studies.

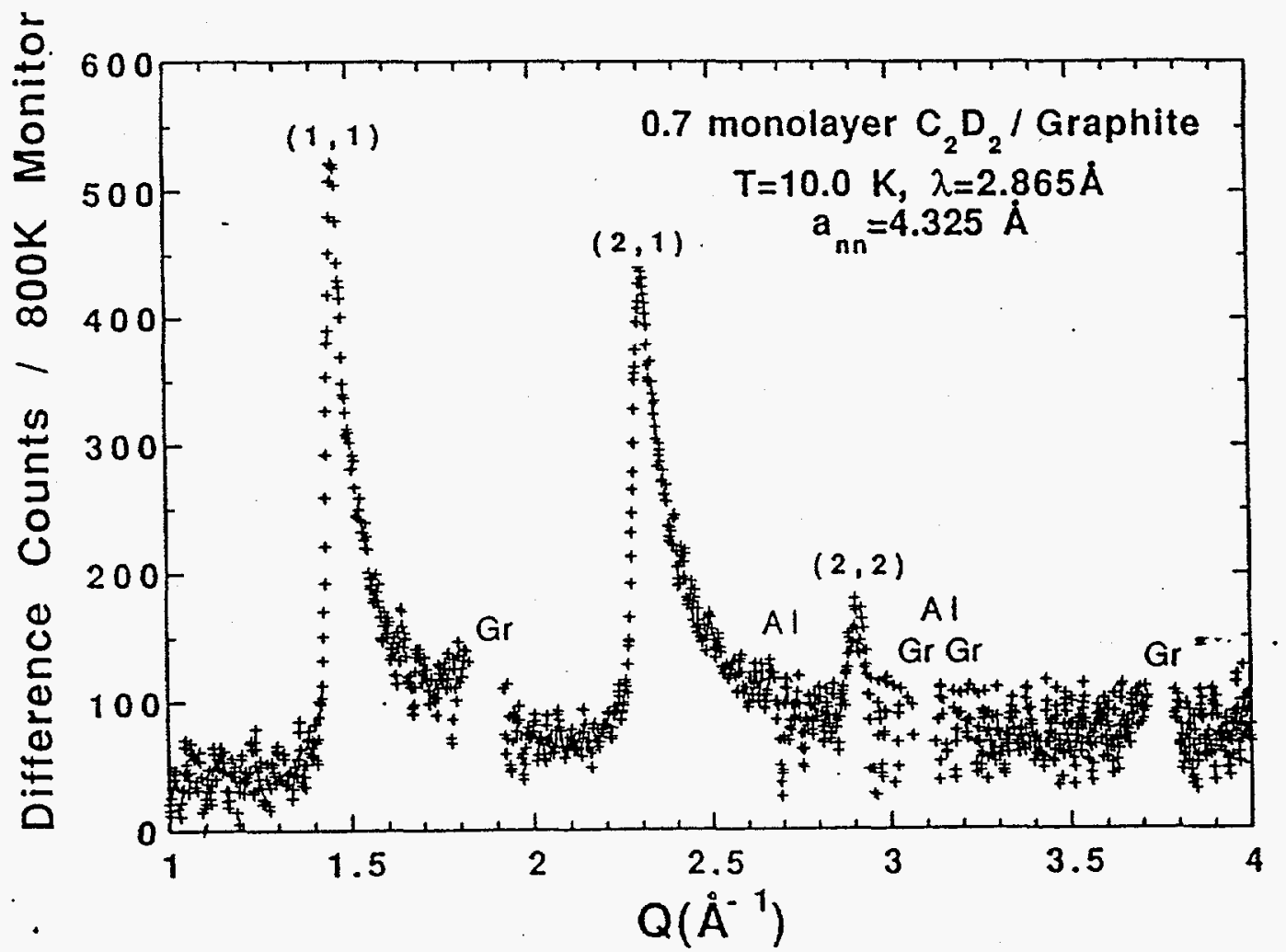

Fig. 4 Difference spectrum from 0.7 monolayer of $\mathrm{C}_{2} \mathrm{D}_{2}$ adsorbed on graphite measured at $\mathrm{T}=10 \mathrm{~K}$. Gr denotes the location of graphite Bragg peaks of the substrate and $\mathrm{Al}$, the location of Aluminum Bragg peaks of the sample cell (from J. Z. Larese et al. (unpublished)). 
Nuclear Physics $(H I-B)$ : The $(\mathrm{n}, \gamma)$ facility on $\mathrm{H} 1-\mathrm{B}$ uses filters to select out a broad range of neutron energies from the thermal regime with a Bi filter, to $2 \mathrm{keV}$ with a Sc filter and up to $24 \mathrm{keV}$ using $\mathrm{Fe}$ and $\mathrm{Al}$ filters. The 3-axis pair spectrometer is used to detect the primary $\gamma$-rays from the $(n, \gamma)$ reaction. Most of the experiments at this facility use the Average Resonant Capture (ARC) technique which guarantees that all levels with certain energy and spin regimes will be observed. This is vital in testing nuclear models by instantly revealing the need for additional degrees of freedom and excitations or by disclosing an excess of these. The ARC studies performed over the last several years have led to the discovery of two of the three dynamical symmetries predicted by the Interacting Boson Approximation (IBA) and, thus, to its confirmation. The $\mathrm{IBA}$ is now the accepted model in nuclear theory.

Chemical Crystallography (H6-M,H6-S): Beam port $\mathrm{H} 6$ consists of two independent instruments used primarily for single crystal elastic studies. H6-M has an analyzer axis and can be used in a three-axis mode if desired. H6-M also has the capability of varying the incident energy whereas H6-S has a fixed monochromator scattering angle providing neutrons with $\lambda=1.16 \AA$ with a $\mathrm{Ge}(220)$ monochromator. The flux at the monochromator positions is $-5.0 \mathrm{x}$ $10^{9} \mathrm{n} / \mathrm{cm}^{2}$-sec. Both spectrometers are equipped with computer controlled, large diameter 4 circle goniometers which allows complete freedom in orienting single crystal samples. An extensive library of operating programs provides automatic crystal centering, unit cell and orientation matrix refinement, and collection of diffraction intensities by the step scan method. The spectrometer control computer is connected to the BNL local area network so that the data are readily accessible for processing from hosts both on- and off-site.

A wide variety of crystal structures have been determined on these instruments. A major emphasis has been studies of the role of hydrogen in chemical bonding in solids since the neutron is a unique probe in determining hydrogen positions. One recent study involved the structure determination of clathrate hydrates, specifically cubic II clathrate $3.5 \mathrm{Xe} \cdot 8 \mathrm{CCl}_{4} \cdot 136 \mathrm{D}_{2} \mathrm{O}$. In this compound the hydrogen bonded water framework encapsulates the $\mathrm{CCl}_{4}$ guest molecules. The structure was refined based upon Bragg intensity data obtained at two different temperatures, 13 and $100 \mathrm{~K}$. Symmetry adapted spherical harmonic wave functions were used to describe the probability density of the disordered $\mathrm{CCl}_{4}$ molecules. By comparing the $\mathrm{CCl}_{4}$ chlorine scattering density at the two temperatures it is established that the $\mathrm{CCl}_{4}$ disorder is primarily dynamic in nature. The method of structure analysis using cubic harmonics is proving to be of great utility in the analysis of other rotationally disordered structures. In fact, recent $\mathrm{x}$-ray studies performed on single crystals of $\mathrm{C}_{60}$ (Buckminster fullerene) have used this analysis to describe the high temperature structure where the carbon clusters are orientationally disordered.

Structural Biology (H3-A, H3-B, H9-B): There are three instruments used primarily for structural biology. H3-A is a neutron spectrometer for protein crystallography. The primary monochromator looks at a thermal beam through tapered collimator which maximizes flux and keeps the background at a minimal level. A focussing PG(002) monochromator is used and the monochromator scattering angle can be varied to give a choice of wavelengths. Higher order neutrons can be eliminated by either a PG filter or a multilayer monochromator which is placed between the primary monochromator and the sample. This latter device can also be used to focus the beam on the sample. The neutron flux at the sample position is $\sim 3 \times 10^{7} \mathrm{n} / \mathrm{cm} 2$-sec. The sample area features a 4-circle diffractometer with a two-dimensional position sensitive detector which has an $80 \%$ efficiency. The Rayleigh resolution is $1.1 \mathrm{~mm}$ (FWHM) and the maximum counting rate is $3 \times 10^{5} \mathrm{cts} / \mathrm{sec}$. The data acquisition computer system controls the diffractometer, processes the counter data and permits on line interactive display. The observed reflections can be processed on line and subjected to the usual crystallographic procedures.

This instrument has been used to elucidate $\mathrm{H}$ bonding in a number of proteins like myoglobin, subtilism, trypsin, and plastocyanin. In addition, detailed studies of bound and 
"solvent" water have been made. The studies of membrane and membrane-drug interaction as well as ordered bacterial phages have been performed.

H3-B is an intermediate resolution SANS station which looks at the thermal part of the reactor spectrum. The double monochromator uses multilayer devices and will operate over a wavelength range of 2.0 to $4.5 \AA$ with an adjustable wavelength band-width $3 \%<\Delta \lambda \lambda<15 \%$. The beam size at the sample is $1 \times 2 \mathrm{~cm}^{2}$ and the scattering angle can be varied between $5^{\circ}<20<40^{\circ}$. An automatic linear sample changer is available as well as an auxiliary cradle. The two dimensional $20 \times 20 \mathrm{~cm}^{2}$ position sensitive detector can be positioned between 0.5 and 2.5 $\mathrm{m}$ behind the sample and has a resolution of $1.3 \mathrm{~mm}$ (horizontal) and $2.5 \mathrm{~mm}$ (vertical). The $\mathrm{Q}$ range accessible is $0.01<Q<3.0 \AA$. A time slicing processor with a switching time of a few microseconds and a computer system for on-line analysis and display of data and results is available.

The instrument is designed for the study of partially ordered systems such as stacked biological membranes and model membranes. It may also be used for solution scattering studies of small molecules, micelles and vesicles.

H9-B is a small angle neutron scattering (SANS) instrument situated at the cold source. It uses a Be-filtered beam emerging from $\mathrm{Ni}$-coated neutron guides with a cross-sectional area of $2.5 \times 5.1 \mathrm{~cm}^{2}$. The beam is monochromatized by interchangeable $\mathrm{Ni}-\mathrm{Ti}$ or $\mathrm{Fe}-\mathrm{Mn}$ multilayers with bilayer spacings between 50 and $100 \AA$ providing a wavelength spread between 6 and $15 \%$. The beam is then collimated over a length of $2 \mathrm{~m}$ by a series of apertures of decreasing diameter. The flux at the sample position with $\lambda=5 \AA$ and a $6 \mathrm{~mm}$ beam is $2 \mathrm{x}$ $10^{6} \mathrm{n} / \mathrm{cm}^{2}$-sec. A temperature controlled sample changer is available for automated measurement of several sample solutions in spectroscopic cells. The sample table is also compatible with other HFBR ancillary equipment such as refrigerators, cryostats, magnets and furnaces. The large area detector $\left(50 \times 50 \mathrm{~cm}^{2}\right)$ is used to record scattering patterns on a $128 \mathrm{x}$ 128 pixel grid and has a resolution of $2.5 \mathrm{~mm}$. It can be placed between 0.5 and $2.0 \mathrm{~m}$ behind the sample and can move about the sample axis on a $45^{\circ}$ arc. The minimum $Q$ obtainable with $\lambda=5.0 \AA$ and a $2-\mathrm{m}$ detector distance is $0.002 \AA-1$. A Micro-VAX computer with a large external time-slicing memory is used for experimental control and data analysis.

The instrument is used primarily for small angle scattering studies on macromolecular biological structures such as protein complexes like the ribosome, and chromosome complexes like the histone. It has also been extensively used to study materials problems such as polymers, porous media, defects in solids, and magnetic and superconducting phenomena.

Figure 5 shows a SANS measurement of intensity vs scattering vector $(s=\sin \theta / \lambda)$ obtained on H9B from a solution of tropomyosin molecules bound in long-pitch helical grooves in actin filaments. These filaments, which constitute the backbone structure of muscle thin filaments, were rendered 'invisible' by perdeuteration and by use of $\mathrm{D}_{2} \mathrm{O}$ buffers. The scattered intensity after buffer subtraction (data points) is due to the tropomyosin double helix with a known pitch of $770 \AA$. The solid line is a least-squares fit to the data revealing the unknown inter-strand separation to be $79 \AA$.

Neutron Irradiation at the HFBR: Irradiation experiments at the HFBR play an important role in the overall research program. The thermal or slow neutrons are used primarily for neutron activation analysis ("finger printing" unknown substance by artificially induced radioactivity) and for producing special purpose radioisotopes. Due to the high neutron flux available, it is possible to produce highly radioactive samples from small quantities of material (high specific activity), and to produce certain isotopes in quantities unattainable by any other practical means. The most unique feature is the availability of the irradiation facilities at the center of the core. This provides a very high flux with a nearly equal amount of fast and thermal neutrons. Fast neutron irradiations are an important tool for the study of radiation damage in materials.

An example of the use of the irradiation facility is the production of a positron beam. A copper sphere of about $4 \mathrm{~mm}$ diameter is inserted into one of the core edge thimbles and after two days of irradiation, a positron emitting source $\left({ }^{64} \mathrm{Cu}\right)$, which has a half-life of 12.8 hours 
and an activity of 20 Curies of positrons, is produced by the nuclear reaction, ${ }^{63} \mathrm{Cu}+\mathrm{n} \rightarrow$ ${ }^{64} \mathrm{Cu}+\lambda(\sigma=4$ barns). Presently, a consortium of physicists from BNL, universities, and industry is using this facility to produce a neutral beam of positronium atoms. Other uses of the high flux neutron facility include the production of radioisotopes for various medical purposes.

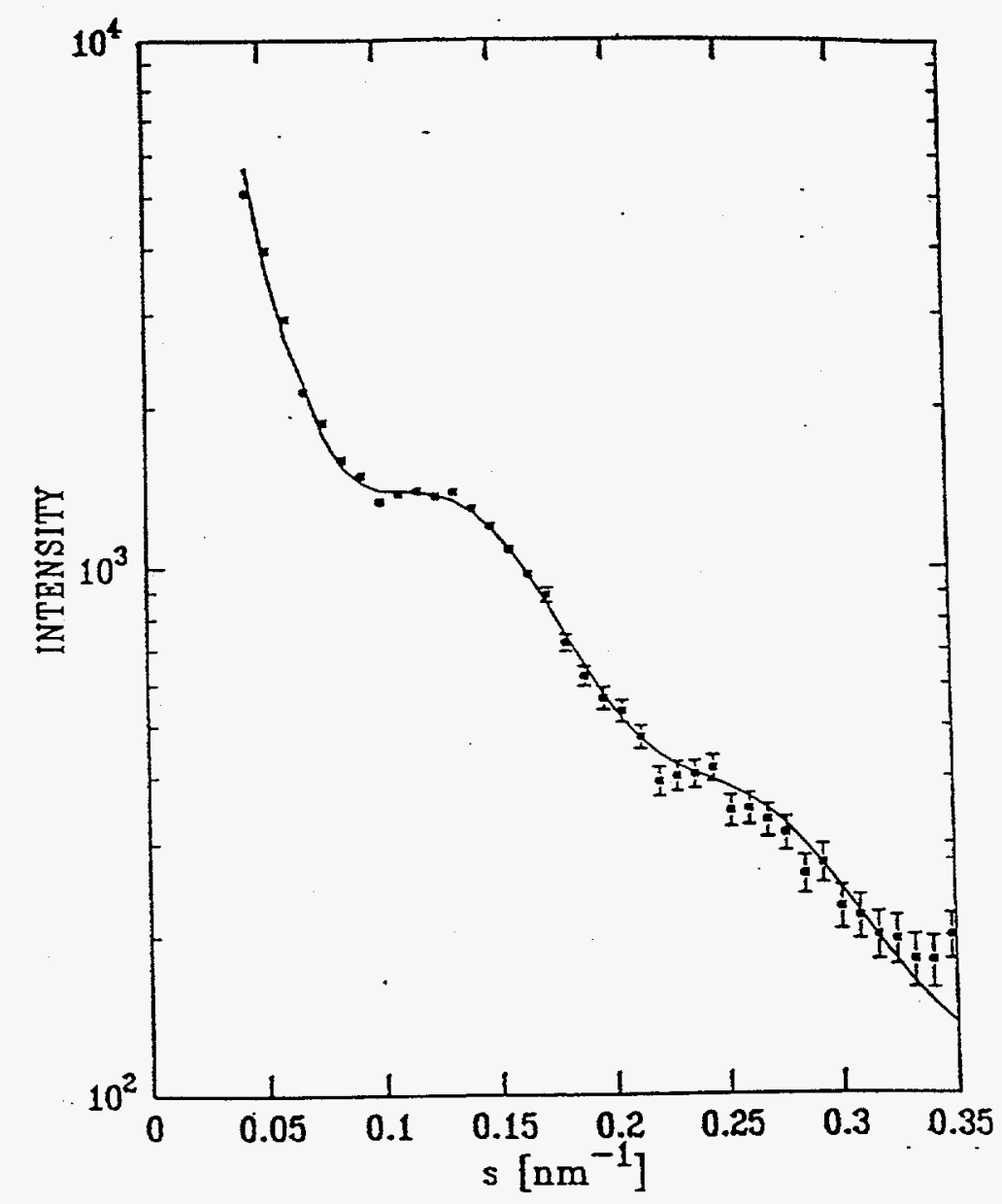

Fig. 5 SANS intensity vs scattering vector, $s=\sin \theta / \lambda$, measured on H9B from a solution of tropomyosin molecules bound in long-pitch helical grooves in actin filaments. The broad peaks correspond to the inter-strand separation of $79 \AA$, (from R. A Mendelson et al., Adv.Biophys. 27, 143 (1991)).

New Instrumentation

Two new instruments have been commissioned at the HFBR. They are the Reflection Spectrometer situated at the $\mathrm{H} 9$ cold source and the high resolution power diffractometer located 
at the H1-A. Both instruments are operated by Participating Research Teams with major participation from external users.

Neutron Reflection Spectrometer $\left(H 9-A^{\prime}\right):$ The monochromatic beam is produced by a PG $(002)$ crystal located in the beryllium-filtered, cold neutron beam immediately downstream of the first monochromating crystal of the H9-A triple axis spectrometer. The beam is deflected by the crystal up to a second PG crystal where it is then deflected horizontally through an evacuated flight tube to a third PG crystal. This latter crystal deflects the beam down onto the horizontal sample surface. Beam shaping is done partly in the flight tube and partly by a pair of computer-controlled slits located between the deflector crystal and the sample. The highly collimated beam is $5 \mathrm{~cm}$ wide, of variable height $(0-5 \mathrm{~mm})$, and has a wavelength of $4.16 \AA$. Samples are mounted on a table which can be manually translated both parallel and perpendicular to the beam and vertically scanned by computer. An active vibration-damping system is available to minimize ripples on the surface of a liquid sample. A well shielded ${ }^{3} \mathrm{He}$ detector measures the intensity of neutrons reflected from the sample surface. Incident beam height and tilt angle, sample height, exit beam tilt angle and detector height are under computer control. Scans can be made at the users option with Q either perpendicular (specular) or in the surface-parallel direction (off-specular). Optimal specular scanning is achieved by varying the beam height to maintain a constant projected beam area on the sample surface. In most cases, the practical scan range is from about 0.002 to $0.25 \AA^{-1}$. The instrument is capable of measuring reflectivities over about six decades.

This instrument incorporates a horizontal reflecting plane and is designed to probe density distributions normal to liquid-vapor, solid-vapor and in certain cases, liquid-solid interfaces. The density profiles are deduced from the $Q$ dependence of the reflected intensity.

High Resolution Neutron Powder Diffractometer (HI-A): This instrument is designed to provide a higher resolution $\left(\Delta \mathrm{d} / \mathrm{d}=5 \times 10^{-4}\right)$ than is currently available in the United States. The resolution is tailored to be highest at large scattering angles, where the Bragg peak density is greatest. This is achieved by a careful matching of collimation and large monochromator scattering angle which is fixed at $120^{\circ}$. The monochromator will be the Ge (511) reflection and composed of a number of thin strips mounted like a 'venetian-blind' which will provide a focused beam on the sample. The wavelength will be $\lambda=1.9 \AA$. The detector assembly consists of 64 film collimators of $5 \mathrm{~min}$ resolution each backed by a ${ }^{3} \mathrm{He}$ counter. The collimators are on 2.5 degree centers, covering a total of 160 degrees. This instrument will be dedicated to structural studies of polycrystalline materials of interest in solid state physics, solid state chemistry, materials science, and mineralogy. It will make feasible structural determinations on materials with large unit cells, and will extend the possibilities for $a b$ initio structure determinations, the study of micro-strain and small crystallite size effect into new areas.

\section{Future Plans}

The HFBR is continuing to modernize its existing user instruments and to add new instruments to meet the needs of the user community. In summer 1995, we will commission a versatile neutron 'optical bench,' which will operate as a dedicated national user facility for peer-reviewed development of improved monochromators, polarizers, detectors and other neutron 'optical' components. In mid-1996 we will complete a restructuring of the H6 beam port by adding super mirror guides optimized for thermal neutrons. This will allow us to improve the two existing single-crystal diffractometers while adding a new medium resolution powder diffractometer and an improved reflectometer. The end station on the thermal guide will be available for future expansion. 
The prospects for the timely funding of the Advanced Neutron Source appear to be waning. We believe it is essential to examine a range of alternative options for providing neutron capabilities into the next century. We believe that the HFBR is capable of reliable operation for several more years without major replacements. If the ANS is indefinitely delayed, a further attractive option would be to refurbish the HFBR by replacing the reactor vessel. (Institut Laue-Langevin recently replaced their vessel at a cost of about \$60M). At the same time, we could add an improved cold-neutron moderator and guide hall, which would provide a facility roughly equivalent to that at ILL. A preliminary technical feasibility study, carried out by a major engineering consultant firm, suggest that the project could be accomplished from scratch within approximately 4-1/2 years, with the reactor out of operation for about two years.

\section{User Policy and Participating Research Teams (PRT)}

The HFBR is open to the world-wide scientific community for high quality fundamental research. This research should be conducted with a minimum of formal administrative interaction and with maximum room for spontaneous developments. To achieve these goals all instruments are managed, developed and maintained by Participating Research Teams (PRTs). A PRT is a group of qualified scientists with common research interests and include scientists from BNL, other DOE, university, and industrial laboratories. In return for its management of the instrument, the PRT is given priority use of the instrument for a fraction of the scheduled beam time. Outside users, i.e. those who are not members of the PRT, must submit proposals which are reviewed and judged on the basis of their scientific merit. Each PRT allocates the remaining beam time (in some cases up to 50\%) to the accepted proposals and provides the necessary technical assistance to the outside user. For more information and proposal forms, please contact Ms. R. Greenberg, Physics Building-510A, Brookhaven National Laboratory, Upton, NY 11973.

Brookhaven National Laboratory is operated for the U. S. Department of Energy, Division of Materials Sciences under Contract No. DE-AC02-76-CH00016 with Associated Universities Incorporated. 